\title{
Comparison of Chest X-Ray Findings of Smear Positive and Smear Negative Patients with Pulmonary Tuberculosis
}

\author{
Azadeh Ebrahimzadeh ${ }^{1}$; Mahyar Mohammadifard ${ }^{2,} ;$ Ghodratallah Naseh $^{3}$ \\ ${ }^{1}$ Department of Internal Medicine, Faculty of Medicine, Birjand University of Medical Sciences, Birjand, Iran \\ 2 Department of Radiology, Imam Reza Hospital, Birjand University of Medical Sciences, Birjand, Iran \\ 3 Department of Surgery, Imam Reza Hospital, Birjand University of Medical Sciences, Birjand, Iran \\ ${ }^{*}$ Corresponding author: Mahyar Mohammadifard, Imam Reza Hospital, School of Medicine, Birjand University of Medical Sciences, Birjand, Iran. Tel: +98-5118414499, Fax: +98- \\ 5612226898, E-mail: mahyarmohammadifard@yahoo.com
}

Received: July 13, 2013; Revised: April 20, 2014; Accepted: June 1, 2014

\begin{abstract}
Background: Tuberculosis is a chronic pulmonary infectious disease that has affected one-third of the people in the world. It causes nine million new cases and two million deaths per year. Chest radiography associated with Ziehl-Neelsen acid-fast staining procedure significantly helps the diagnosis of pulmonary tuberculosis (PTB). Chest radiography can help the diagnosis of tuberculosis in patients with a negative smear sample result that is mainly diagnosed with delay.

Objectives: In this study, chest X-ray findings of PTB were compared in two groups of smear positive and smear negative patients.

Patients and Methods: In this retrospective descriptive-analytical study, 376 patients who had been confirmed with PTB were referred to Birjand Health Care Center from 2001 to 2006. Out of the 376 patients, 100 patients with a positive smear based on WHO criteria were selected. In addition, among negative smear patients, 100 were selected in whom similar demographic characteristics with positive smear patients were seen. All of them had undergone chest radiographies that were then interpreted by two expert radiologists independently. Moreover, all patients' sputa were examined by an expert laboratory technician at the reference laboratory of the health center. The obtained data were analyzed by means of frequency distribution table and descriptive statistics using SPSS (version 15) and Chi-square statistical test.

Results: Except reticulo-nodular infiltration, the relative frequency of other radiographic findings in positive smear patients were more than negative smear patients; and only differences in calcification variables, mediastinal widening, patchy infiltration and hilar adenopathy were statistically significant $(\mathrm{P}<0.05)$.

Conclusions: Based on the results of this study, although radiographic findings are not diagnostic in PTB, they are helpful if the assessment associates with the view of clinical manifestations and sputum smears.
\end{abstract}

Keywords: Pulmonary Tuberculosis; Smear Positive; Smear Negative; Radiological Findings

\section{Background}

Tuberculosis is a chronic lung infection that is caused by mycobacterium germs. This disease caused more than $30 \%$ of all deaths in the 19 th and 20 th centuries and has currently infected one-third of all people worldwide. It causes nine million new cases and two million deaths annually (1-3). TB is the second most fatal infectious agent after AIDS. Eighty percent of all tuberculosis patients reside in 22 countries primarily in the thirdworld $(1,2)$. It has been estimated that over one billion people will be infected by tuberculosis by 2020 and the number of patients will reach 200 million. In addition, one million deaths will occur due to tuberculosis. At present, 30 million people are infected by tuberculosis, while it is estimated that almost 300 million people are contaminated and about 90 million would be involved with tuberculosis within the next ten years $(1,4,5)$.

The prevalence of tuberculosis reduced to $5 \%$ annually until 1995 (6), but within the next five years, the prevalence increased to $15.8 \%$. Currently, tuberculosis in as- sociation with acquired immunodeficiency syndrome (AIDS) is the most important public health problem in Asia, Africa and Latin America. Hence, it was called an emergency by WHO in $1993(6,7)$.

The best and most definitive method in the diagnosis of pulmonary tuberculosis (PTB) is sputum smear and Ziehl-Neelsen staining with several consecutive tests (1$3)$. In the recent decades, due to the increase in diversity and number of diagnostic techniques, smear positive tuberculosis has raised $(2,3)$. Although smear negative tuberculosis has significantly reduced, still a considerable number of cases fall at this group. For example, in Iran, smear positive tuberculosis increased from 3980 to 5430 cases from 1992 to 1996 (7 per 100,000 vs. 8.9 per 100,000); and smear negative tuberculosis reduced from 13182 cases to 6081 (23.1 per 100,000 vs. 10 per $100,000)(8)$.

In a study done in Iran in 1996, 11.25 per 100,000 smear positive and 6.62 per 100,000 smear negative and 2.72

Copyright ( ) 2014, Tehran University of Medical Sciences and Iranian Society of Radiology; Published by Kowsar. This is an open-access article distributed under the terms of the Creative Commons Attribution-NonCommercial 4.0 International License (http://creativecommons.org/licenses/by-nc/4.0/) which permits copy and redistribute the material just in noncommercial usages, provided the original work is properly cited. 
per 100,000 extra-pulmonary tuberculosis were detected in Birjand, South Khorasan, which ranked third place after Systan-Baluchestan and Golestan provinces (9). Since each smear negative patient can contaminate two people each year, rapid diagnosis of tuberculosis is as essentially important as the smear positive patient. In smear negative and even smear positive cases, radiographic changes are helpful in disease diagnosis. Although some chest X-ray findings, including nodular or patchy infiltration in the posterior parts of the superior lobes or anterior segments of the inferior lobes particularly in bilateral involvement and associated with cavity, are not specific for pulmonary tuberculosis, clinical manifestations and epidemiologic findings are very diagnostic $(10,11)$. Generally, common radiologic findings in secondary PTB are demonstrated as follows: bronchiectasis, consolidation, patchy, cavitary, and miliary TB $(12,13)$.

\section{Objectives}

This study has been done to compare chest X-ray changes seen in smear positive and smear negative tuberculosis patients.

\section{Patients and Methods}

In this retrospective descriptive-analytical study, 376 patients who had been confirmed with PTB were referred to Birjand Health Care Center from 2001 to 2006. Out of the 376 patients, 100 patients with a positive smear based on WHO criteria were selected. In addition, among negative smear patients, 100 patients were selected as the control group, in whom similar demographic characteristics with positive smear patients were seen.

Based on WHO criteria, positive smear PTB is defined with at least two positive sputum smears of acid-fast bacillus or one positive sputum smear associated either with one positive sputum culture or chest radiographic findings compatible with PTB. Negative smear PTB was confirmed when positive radiographic changes compatible for PTB have been detected and in spite of a 10-14 day wide spectrum antibiotic therapy, no change in the patient's general condition was noted from whom two negative serial sputum smears were taken with at least two weeks interval and in each session, three samples were examined and all samples were negative for acid fast bacillus. In addition, smear negative PTB is defined when a severely ill patient with radiographic changes in favour of PTB lesions (interstitial tissue involvement or miliary TB) shows two negative samples for acid fast bacillus. The physician then decided to start anti-TB treatment in a patient with initially negative sputum smears and a positive sputum culture.

All of the patients underwent chest radiographies that were then interpreted by two expert radiologists independently. Moreover, all patients' sputa were exam- ined by an expert laboratory technician at the reference laboratory of the health center. The obtained data were analyzed by means of frequency distribution table and descriptive statistics using SPSS version 15 for Windows (SPSS Inc., Chicago, Ill, USA) and Chi-square test was used for statistical analysis. P-values less than 0.05 were considered statistically significant.

\section{Results}

Out of the 200 patients who were studied, most of them were Iranian citizens (97\%), 63.5\% were female and $59 \%$ of the cases were from urban areas. The age distribution was $15.5 \%, 45 \%$, and $39.5 \%$ in the age groups of $1-13$, 14-55, and above 55 years old, respectively. The mean age in the females was $50.88 \pm 22.22$ years and $49.11 \pm 22.82$ years in the males $(\mathrm{P}=0.57), 48.31 \pm 22.41$ years in the positive smear group and $51.66 \pm 21.52$ years in the negative smear group $(\mathrm{P}=0.28)$.

Forty six percent of the smear positive and $25 \%$ of the smear negative patients had ESR $>50(\mathrm{P}<0.001)$. Based on the results of this study, the most common radiographic manifestations in smear positive cases were calcification (56\%), adenopathy (53\%), bronchiectasis (40\%), reticulonodular infiltration (36\%), and patchy infiltration (30\%).

Apical involvement, cavity, incomplete pulmonary destruction, pleural effusion, mediastinal widening and fibrosis were the next common findings, while in smear negative patients, calcification (27\%), reticulo-nodular infiltration (41\%), fibrosis (23\%), adenopathy (21\%), and patchy infiltration (14\%) were the most common findings. Likewise, apical involvement, bronchiectasis, pleural effusion, cavity, mediastinal widening were the next common findings. The frequency of patchy infiltration, calcification, mediastinal widening, and hilar pulmonary adenopathy was higher in smear positive patients $(\mathrm{P}=0.006, \mathrm{P}<0.001, \mathrm{P}=0.02$ and $\mathrm{P}<0.001$, respectively). Although the frequency of pleural effusion, complete pulmonary destruction, miliary TB, pulmonary fibrosis and apical involvement were common in smear positive cases and reticulo-nodular infiltration in smear negative ones, but neither showed any significant statistical difference.

So, the frequency of calcification, pulmonary hilar adenopathy, incomplete pulmonary destruction, cavity, mediastinal widening, bronchiectasis, and patchy infiltration were more common in the smear positive groups compared to smear negative groups that were statistically significant (all Ps $<0.05$ ). The frequency of pulmonary fibrosis, apical infiltration, pleural effusions, complete pulmonary destruction and miliary TB were higher but with no significant statistical difference (all Ps $>0.05$ ). In contrast, reticulo-nodular infiltration was seen more commonly in smear negative groups rather than in smear positive groups with no significant statistical difference $(P>0.05)$ (Table 1). 
Table 1. Radiological Changes in Smear Positive and Smear Negative Groups

\begin{tabular}{lccc}
\hline Radiologic Finding & $\begin{array}{c}\text { Smear } \\
\text { Negative, \% }\end{array}$ & $\begin{array}{c}\text { Smear } \\
\text { Positive, \% }\end{array}$ & P Value \\
\hline Calcification & 27 & 56 & $\mathrm{P}<0.001$ \\
\hline Hilar adenopathy & 21 & 53 & $\mathrm{P}<0.001$ \\
Incomplete destruction & 4 & 16 & $\mathrm{P}=0.005$ \\
$\begin{array}{l}\text { Apical infiltration } \\
\text { Widening of }\end{array}$ & 2 & 7 & $\mathrm{P}=0.09$ \\
mediastinum & 2 & 10 & $\mathrm{P}=0.02$ \\
Bronchiectasis & 12 & 40 & $\mathrm{P}=0.001$ \\
$\begin{array}{l}\text { Reticulo-nodular } \\
\text { infiltration }\end{array}$ & 41 & 36 & $\mathrm{P}=0.47$ \\
Patchy infiltration & 14 & 30 & $\mathrm{P}=0.006$ \\
$\begin{array}{l}\text { Lung fibrosis } \\
\text { Cavity }\end{array}$ & 23 & 30 & $\mathrm{P}=0.26$ \\
\hline $\begin{array}{l}\text { Pleural effusion } \\
\text { Complete destruction }\end{array}$ & 22 & 53 & $\mathrm{P}=0.001$ \\
\hline Miliary tuberculosis & 6 & 11 & $\mathrm{P}=0.82$ \\
\hline
\end{tabular}

\section{Discussion}

Despite great advantages in prevention and treatment, PTB is still an important health problem in Iran as well as many other endemic areas $(4,8)$. So, proper diagnosis and follow-up of the patients is essential while due to large geographic areas, and different access levels to health services, approaching more accessible and cheaper imaging and laboratory techniques is welcome(14). Therefore, in this descriptive study, the patients who had been confirmed with PTB were compared in term of radiologic and laboratory manifestations and here our findings are compared with the literature.

In the study conducted by Rathman et al., of the 1389 cases suspicious for tuberculosis, $34 \%$ were smear positive and 66\% were smear negative. Radiographic changes were assessed in the two groups. Cavity was noted in smear positive cases more than smear negative ones (40\% vs. $25 \%)$, ( $>0.001)$. Likewise, calcification was significantly more common in smear positives than smear negatives $(\mathrm{P}<0.001)(15)$.

In the study performed by van Cleef et al. smear negative and smear positive tuberculosis patients were followed up for ten years and the chest radiographic changes raised from $1 \%$ to $10 \%$ in the smear negative cases. Generalized reticulo-nodular infiltration (55\%); cavity (30\%) and pleural effusion (15\%) were more frequent in smear negative than smear positive cases $(\mathrm{P}<0.001)$. Whilst, patchy infiltration (45\%), calcification (45\%), adenopathy (30\%), and bronchiectasis (22\%) were more frequent in smear positives compared to the smear negative group $(\mathrm{P}=0.001)(16)$.

In the study carried out by Miller et al. pulmonary find- ings compatible with smear positive were patchy infiltration, cavitation and calcification (17). In the mentioned study, pulmonary lesions compatible with smear positive PTB, patchy infiltration, cavitation and calcification were more frequent, while in the study conducted by Gatner et al. pulmonary lesions compatible with smear negative PTB, hilar or mediastinal adenopathy, diffuse reticulonodular infiltration and pleural effusion were more common (18).

In our study, similar to other studies, some pulmonary changes were seen more frequently with a statistically significant difference between the smear positive and smear negative patients such as patchy infiltration, calcification, and adenopathy in smear positive and reticulonodular infiltration in smear negative ones. This might help the diagnosis of the disease and should be kept in mind by physicians, hence, according to the study performed by Jones et al. in jail, $20 \%$ of the patients with smear negative PTB might have been missed when the radiographic changes were neglected (19). Likewise, in another study on 518 PTB patients, $14.8 \%$ of smear negative patients had typical pulmonary findings compatible with PTB (20). In the study carried out by Razaghi et al. on 100 TB patients in Kashan, Iran, the most common radiologic findings were reticulo-nodular infiltration (18\%), bronchiectasis (13\%) and atelectasis (3\%) (21).

In the study performed by Rajabzadeh in Ghouchan, Iran, on TB patients who were older than 50 years old, segmental infiltration was seen in $40 \%$ of the cases and primarily unilateral lung infiltration (83\%) was the most common finding which was followed by apical involvement (30\%), pleural effusion (26\%), cavity (20\%) and adenopathy (16\%) (22). In our study, both smear positive and smear negative patients were evaluated and typical pulmonary changes such as apical involvement and cavity were more common, most likely due to the higher age range of our cases compared to other studies.

In the study conducted by Bakhshayeshkaram et al. on 100 smear positive TB patients from Tehran, the most pulmonary involvements were: reticulo-nodular infiltration (98\%), cavity (60\%), pleural thickening (45\%), adenopathy (36\%) and fibrosis (30\%) (23). In another study on smear positive patients $(n=50)$, infiltration with or without cavity in the upper pulmonary segments (78\%), hilar and mediastinal adenopathy (65\%), and pleural effusion (45\%) were the most common radiologic manifestations (24). The very high percentage of reticulo-nodular infiltration in the study carried out by Bakhshayeshkaram et al. is probably due to the attribution of chronic pulmonary and occupational diseases in the mentioned study that were not excluded. Likewise, in the study performed by Cohen et al., 12 out of 50 PTB cases coincided with malignancy; therefore, a higher frequency of adenopathy and cavity were noted (24).

In our study, similar to other studies, some pulmonary changes were seen more frequently with statistically significant differences between smear positive and smear 
negative patients. Paying attention to these differences by physicians would lead to better diagnosis of the disease. Moreover, in view of the fact that common radiologic findings can be seen in tuberculosis as well as pulmonary malignancy, pulmonary abscess and chronic pulmonary diseases, the best approach is to rule out these diseases in the differential diagnosis before any medical intervention.

Based on the findings and results of our study, clinical manifestations and sputum smear in the light of radiographic changes are very useful tools in the diagnosis of PTB and hence a remarkable help for the treatment of PTB patients.

\section{Acknowledgements}

Hereby we acknowledge Dr. Mohammad M. Mohammadifard for his great support, reporting the radiographs and our colleagues working at the TB unit of Birjand Health Care Center. We also appreciate our valued patients for their cooperation.

\section{Funding/Support}

This study was supported by the Faculty of Medicine and the Chancellery for Research of Mashhad University of Medical Sciences.

\section{References}

1. Raviglion MC, O' Brein RJ. Tuberculosis. In: Fauci A, Braunwald E, Kasper D, Hauser S, Longo D, Jameson J, et al editors. Harrison's Principles of Internal Medicine. 17th ed: Mcgraw-hill; 2008. pp. 1006-21.

2. Harper C, Watt Y, Raviqlion ML. Infectious Diseases, Tuberculosis. In: Cecil RLF, Goldman LW, Ausiello DA editors. Cecil Medicine. 23th ed:W.B. Saunders Elsevier; 2008. pp. 2292-307.

3. Fitzqeraid DW, Sterling TR, Haas DW. Tuberculosis. In: Mandell GL, Bennett JE, Dolin R editors. Mandell, Douglas, and Bennett's principles and practice of infectious diseases. 7th ed: Churchill Livingstone/Elsevier; 2010.

4. Maher D, Raviglione M. Global epidemiology of tuberculosis. Clin Chest Med. 2005;26(2):167-82.

5. Gomes MG, Franco AO, Gomes MC, Medley GF. The reinfection threshold promotes variability in tuberculosis epidemiology and vaccine efficacy. Proc Biol Sci. 2004;271(1539):617-23.

6. Dye C, Watt CJ, Bleed DM, Hosseini SM, Raviglione MC. Evolution of tuberculosis control and prospects for reducing tuberculosis incidence, prevalence, and deaths globally. JAMA. 2005;293(22):2767-75.

7. Harper ME, Hill PC, Bah AH, Manneh K, McAdam KP, Lienhardt C. Traditional healers participate in tuberculosis control in The
Gambia. Int J Tuberc Lung Dis. 2004;8(10):1266-8.

8. Resaii AR, Hendesi F, Rezvani SM, editors. Epidemiology of tuberculosis in Gillan (1996-2006).; 18th national congress on tuberculosis.; 2007; Sanandaj, IR Iran. p.1.

9. Ebrahimzadeh A, Eshaghi S, Bahlgerdi M, Hashemzehi M, editors. Tuberculosis epidemiology in South Khorasan in 2000-2006.; 18th national congress on tuberculosis.; 2007; Sanandaj, IR Iran. p. 218.

10. van Cleeff MR, Kivihya-Ndugga LE, Meme H, Odhiambo JA, Klatser $\mathrm{PR}$. The role and performance of chest X-ray for the diagnosis of tuberculosis: a cost-effectiveness analysis in Nairobi, Kenya. BMC Infect Dis. 2005;5:111.

11. Rafii S, Sedaghat M, Anvari R, Hessam H, Nosrati HR, Mofidi AM editors. Smear positive pulmonary tuberculosis in Golestan.; 18th national congress on tuberculosis.; 2007; Sanandaj, IR Iran. p. 5.

12. McCray E, Weinbaum CM, Braden CR, Onorato IM. The epidemiology of tuberculosis in the United States. Clin Chest Med. 1997;18(1):99-113.

13. Martin G, Lazarus A. Epidemiology and diagnosis of tuberculosis. Recognition of at-risk patients is key to prompt detection. Postgrad Med. 2000;108(2):42-4-53-4.

14. Marciniuk DD, McNab BD, Martin WT, Hoeppner VH. Detection of pulmonary tuberculosis in patients with a normal chest radiograph. Chest.1999;115(2):445-52.

15. Rathman G, Sillah J, Hill PC, Murray JF, Adegbola R, Corrah T et al. Clinical and radiological presentation of 340 adults with smear-positive tuberculosis in The Gambia. Int J Tuberc Lung Dis. 2003;7(10):942-7.

16. van Cleeff MR, Kivihya-Ndugga L, Githui W, Nganga L, Odhiambo J, Klatser PR. A comprehensive study of the efficiency of the routine pulmonary tuberculosis diagnostic process in Nairobi. Int Tuberc Lung Dis. 2003;7(2):186-9.

17. Miller WT, MacGregor RR. Tuberculosis: frequency of unusual radiographic findings. AJR Am J Roentgenol. 1978;130(5):867-75.

18. Gatner EM, Burkhardt KR. Correlation of the results of X-ray and sputum culture in tuberculosis prevalence surveys. Tubercle. 1980;61(1):27-31.

19. Jones TF, Schaffner W. Miniature chest radiograph screening for tuberculosis in jails: a cost-effectiveness analysis. Am J Respir Crit Care Med. 2001;164(1):77-81.

20. Hernandez-Garduno E, Cook V, Kunimoto D, Elwood RK, Black WA, FitzGerald JM. Transmission of tuberculosis from smear negative patients: a molecular epidemiology study. Thorax. 2004;59(4):286-90.

21. Razaghi R, Talari HR, Akbari H, Lotfii A, editors. Chest X-ray findings in elderly pulmonary tuberculosis patients that reffered to Kashan center control of disease 1996-2006.; 18th national congress on tuberculosis.; 2007; Sanandaj, IR Iran. p. 20.

22. Rajabzadeh A, editor. Clinical and ragiological finding in pulmonary tuberculosis patients above 50 years. .; 13th national congress on tuberculosis ..1997; Tehran, IR Iran.

23. Bakhshayesh KM, Masjedi MR, Ashabi H, Kiani F. Chest X_ray findings in smear positive pulmonary tuberculosis patients. J Trop Infec Dis .1996;1:24-8.

24. Cohen R, Muzaffar S, Capellan J, Azar H, Chinikamwala M. The validity of classic symptoms and chest radiographic configuration in predicting pulmonary tuberculosis. Chest.1996;109(2):420-3. 